\title{
Mapping quantitative trait loci for reproduction in pigs
}

\author{
S.C. Hernandez, H.A. Finlayson, C.J. Ashworth, C.S. Haley \\ and A.L. Archibald
}

The Roslin Institute and Royal (Dick) School of Veterinary Studies, University of Edinburgh, Roslin, Midlothian EH25 9PS, Scotland, UK

Reproductive performance is a critical component of sustainable animal production systems. The low heritability of reproductive performance traits such as litter size, ovulation rate, and prenatal survival and their expression only in females limits improvement of these traits through traditional selective breeding programs. However, there is abundant evidence of genetic variation in these traits between pig breeds, which could be exploited to improve reproductive performance through selective breeding. The Chinese Meishan breed is one of the most prolific pig breeds known, displaying greater litter size than commercial Western breeds, such as Large White, through higher levels of prenatal survival for a given ovulation rate. But Meishan pigs have poor growth rates and high carcass fat content. However, increasing the number of viable and productive offspring per reproductive female reduces financial and environmental costs and improves the sustainability of the system. Thus, the superior Meishan alleles for reproduction traits are potentially commercially valuable. As only a fraction of the genes / loci that underpin the Meishan's superior reproductive performance have been identified to date, it is evident that the genetics of reproductive performance merits further investigation. In an earlier study we mapped a QTL (quantitative trait loci) with effects on embryo survival and litter size to the distal end of pig chromosome 8 (King et al. 2003). The objective of this study is to identify QTL affecting ovulation rate, teat number, litter size, number born alive and embryo survival, and characterize candidate gene(s) underlying such QTL.

Our strategy to identify genetic markers for reproduction traits combines identifying QTL (regions of the genome linked to the phenotypes) through genome scans using interval mapping and testing genes recognized as candidates on both positional and physiological grounds. The QTL analyses involve testing for associations between variation in the trait(s) of interest and the inheritance of chromosomal segments from the parental animals. The inheritance of chromosomal segments through the QTL mapping population is tracked by genotyping the population for polymorphic genetic markers - microsatellites and single nucleotide polymorphisms (SNPs). The three-generation Roslin Institute Meishan x Large White F2 QTL mapping population was genotyped for ten additional markers across the QTL found previously on chromosome 8 and for 127 markers evenly spaced across the rest of the genome. The marker genotypes and trait data were lodged in the resSpecies database (www.resSpecies.org). Linkage maps were constructed using Multimap and Crimap (Green et al. 1990) and the resulting maps checked for anomalous double recombinants with the chrompic function. Anomalous genotypes were checked and corrected or omitted from the analysis. The marker orders in the linkage map exhibited good agreement with international reference linkage maps. QTL analyses were performed using the "fixed QTL allele" model on the GridQTL portal (www. gridqtl.org.uk) (Seaton et al. 2002). 
A genome-wide scan was conducted for QTL with effects on the following traits: embryo survival, total ovulation rate, total teat number, litter size, and number born alive. This scan revealed QTLs for litter size (total number born) on SSC6 (102 CM), SSC8 (114 cM), SSC13 $(135 \mathrm{cM})$ and SSC18 (37 cM); QTL for number born alive on SSC8 (114 cM); QTL for embryo survival on SSC8 $(135 \mathrm{cM})$ and SSC10 $(111 \mathrm{cM})$ and QTL for total ovulation rate on SSC7 $(56 \mathrm{cM})$, SSC13 $(38 \mathrm{cM}), \operatorname{SSC} 15(8 \mathrm{cM})$, and SSC18 (37 cM), and for total teat number on SSC5 (57 cM), SSC6 $(20 \mathrm{cM})$ and SSC18 $(0 \mathrm{cM})$. These results were used as background effects in further analyses to confirm the presence or absence of these QTL and to potential reveal additional QTL. This new scan revealed QTL for litter size (total number born) on SSC8 $(114 \mathrm{cM})$ and SSC13 (35 cM), confirming two of the previous ones; QTL for number born alive on SSC13 (41 cM); QTL for embryo survival on SSC8 $(135 \mathrm{cM})$, and QTL for total ovulation rate on SSC7 (9-13 cM), SSC13 $(39 \mathrm{cM})$, and SSC14 $(21 \mathrm{cM})$, and for total teat number on SSC5 (56 cM), SSC1 $1(0 \mathrm{CM})$ and SSC12 $(3 \mathrm{CM})$. These QTL require further studies to confirm the effect on the different traits. As noted above, QTL with effects on litter size and embryo survival had been discovered co-located at the distal end of chromosome 8 in an earlier study (King et al. 2003). The fine mapping of these QTL enabled by genotyping the population for additional markers revealed two QTL locations - a QTL for embryo survival on chromosome 8 (SSC8) at $134 \mathrm{cM}$, a QTL for litter size at position $114 \mathrm{cM}$, together with a QTL with effects on the number born alive. The emerging pig genome sequence and the homologous regions of the human and mouse genome sequences will be inspected for potential positional and comparative positional candidate genes. The SPP1 (Secreted phosphoprotein 1) gene, which is known for its role in communication between the developing embryo and the sow, with a key role in conceptus implantation and maintenance of pregnancy, is a strong candidate gene as it is located under the peak of the SSC8 QTL with effects on embryo survival.

The resolution for the QTL found previously has been improved by the addition of a further ten markers across the region of interest. The genome scan revealed twelve QTLs of which only two are annotated in the pigQTLdb (www.animalgenome.org/QTLdb/pig.htmi). Future studies will involve fine mapping the QTL revealed by the genome scan and prioritisation of positional candidate genes underlying the QTL for functional analyses. For example, candidate genes for embryo survival QTL will be examined for their expression at the RNA and protein levels in relevant tissue samples (feto-placental tissue) from animals of different breeds.

We thank the British Pig Executive (SCH) and BBSRC (ALA, CIA, CSH) for financial support and the Birrel-Gray Travelling Scholarship (SCH) and the Society for Reproduction and Fertility Travel Grant (SCH) for the awards to attend the conference.

\section{References}

Green P, Falls K, \& Crooks S 1990 Documentation for CRI-MAP, Version 2.4. St.Louis, Mo., Washington Univesity School of Medicine.

King AH, .Jiang Z, Gibson JP, Haley CS, \& Archibald AL 2003 Mapping quantitative trait loci affecting female reproductive traits on porcine chromosome 8. Biology of Reproduction 68 2172-2179.

Seaton G, Haley CS,.Knott SA, Kearsey M, \& Visscher PM 2002. QTL Express: mapping quantitative trait loci in simple and complex pedigrees. Bioinformatics. 18 339-340. 